\title{
Small world networks and creativity in audio clip sharing
}

\section{Gerard Roma}

Music Technology Group, Universitat Pompeu Fabra

08018 Barcelona, Spain

E-mail: gerard.roma@upf.edu

\section{Perfecto Herrera}

Music Technology Group,Universitat Pompeu Fabra

08018 Barcelona, Spain

E-mail: perfecto.herrera@upf.edu

\section{Massimiliano Zanin}

Centre for Biomedical Technology,Polytechnic University of

Madrid

28223 Pozuelo de Alarcón, Madrid, Spain

E-mail: massimiliano.zanin@ctb.upm.es

\section{Sergio L Toral}

E. S. Ingenieros, University of Seville

41092, Sevilla, Spain

E-mail:toral@esi.us.es

\section{Frederic Font}

Music Technology Group, Universitat Pompeu Fabra

08018 Barcelona, Spain

E-mail: frederic.font@upf.edu

\section{Xavier Serra}

Music Technology Group, Universitat Pompeu Fabra

08018 Barcelona, Spain

E-mail: xavier.serra@upf.edu

\begin{abstract}
Sharing communities are changing the way audio clips are obtained in several areas, ranging from music to game design. The motivations for people to record and upload sounds to these sites are likely to be related to social factors. In this paper we describe several
\end{abstract}


networks that can be extracted from user activities in these systems. We propose the notion of creativity as an objective for this kind of community, and how some indicators of creativity can be extracted. We investigate the relationship between the network properties and the creative outcome indicators through an empirical analysis of data from Freesound.org, a widely used website for sharing audio clips.

Keywords: audio clip sharing; social networks; creativity.

\section{Reference}

\section{Biographical notes:}

Gerard Roma received a degree in philosophy from Universitat Autònoma de Barcelona (UAB) in Barcelona, Spain, in 1997. After several years working in software development he went back to the university and obtained a Master in Information and Communication Technologies from Universitat Pompeu Fabra (UPF) in 2008. He is currently a PhD candidate at the Music Technology Group in UPF, were his research focuses on computational models and applications for supporting collective musical creativity.

Perfecto Herrera received the degree in psychology from the University of Barcelona, Barcelona, Spain, in 1987. He was with the University of Barcelona as a Software Developer and as assistant professor. His further studies focused on sound engineering, audio postproduction, and computer music. Now he is finishing his $\mathrm{PhD}$ on music content processing in the Universitat Pompeu Fabra (UPF), Barcelona. He has been working with the Music Technology Group, (UPF) since its inception in 1996, first as responsible for the sound laboratory/studio, then as a researcher. He worked in the MPEG-7 standardization initiative from 1999 to 2001. Then, he collaborated in the EU-IST-funded CUIDADO project, contributing to the research and development of tools for indexing and retrieving music and sound collections. This work was somehow continued and expanded as scientific coordinator for the Semantic Interaction with Music Audio Contents (SIMAC) project, again funded by the EU-IST. He is currently the Head of the Department of Sonology, Higher Music School of Catalonia (ESMUC), where he teaches music technology and psychoacoustics. His main research interests are music content processing, classification, and music perception and cognition.

Massimiliano Zanin received the degree in Aeronautical Management from the Universidad Autónoma de Madrid, Madrid, Spain, in 2010. He is now working as a Complex Systems researcher in the Centre for Biomedical Technology, applying techniques adapted from Complex Networks analysis to the study of biomedical problems.

S.L. Toral received his $\mathrm{MS}$ and $\mathrm{PhD}$ in Electrical and Electronic Engineering from the University of Seville, Spain, in 1995 and 1999, respectively. He is currently an Associate Professor at the Department of Electronic Engineering, US. His main research interests include realtime and distributed systems, Ad Hoc networks, open source software projects, and embedded operating systems. 
Frederic Font received a three-year degree in telecommunications from Universitat Politècnica de Catalunya (UPF), Barcelona, Spain, in 2007. He obtained a Master in Sound and Music Computing from Universitat Pompeu Fabra (UPF), Barcelona, Spain, in 2010. He is currently enrolled in a $\mathrm{PhD}$ program at the Music Technology Group (UPF). His research interests include the characterization of communities of users in online sound and music sharing sites to support musical creativity.

Xavier Serra is Associate Professor of the Department of Information and Communication Technologies and Director of the Music Technology Group at the Universitat Pompeu Fabra in Barcelona. After a multidisciplinary academic education he obtained a $\mathrm{PhD}$ in Computer Music from Stanford University in 1989 with a dissertation on the spectral processing of musical sounds that is considered a key reference in the field. His research interests cover the understanding, modelling and generation of musical signals by computational means, with a balance between basic and applied research and approaches from both scientific/technological and humanistic/artistic disciplines. Dr. Serra is very active in promoting initiatives in the field of Sound and Music Computing at the local and international levels, being involved in the editorial board of a number of journals and conferences and giving lectures on current and future challenges of the field. He has recently been awarded an Advanced Grant of the European Research Council to carry out the project CompMusic aimed at promoting multicultural approaches in music computing research.

\section{Introduction}

Audio clips are building blocks for many creative processes. From computer games, interactive media and user interfaces to music and film, it is very common to use audio clips, either tailored for the occasion or conveniently provided as libraries by some publisher. Even if coming from a library, these sounds can be manipulated at will to suit a creative purpose.

Unsurprisingly, internet is becoming the dominant distribution medium for such files. Perhaps overshadowed by the transformation of the music industry, the market of sound libraries has faced similar issues and became intermingled with personal file sharing, to the extent that the licensing conditions for royalty-free sounds inherited from the pre-internet era have been questioned (Lessig, 2008).

Users are now able to organize and share sound clips produced by themselves through the web, usually in a less organized manner than the traditional editorial processes of library publishers. A growing number of sites offer royalty-free sounds under a variety of licenses, often for free. Perhaps more interestingly, some of them are organized so that users can contribute sounds as well as download them. This process is very similar to more common activities such as sharing text, images or videos, but it is less known to the general public. Unlike most multimedia files shared through on websites, sound clips are not simply enjoyed online, but downloaded and used as building blocks for the mentioned creative processes. Thus, the process 
of sharing sound clips is associated with a certain cultural and economic value, also similar to other community-driven resources such as Wikipedia.

With the generalization of content management systems and the focus on usergenerated media that characterized the Web 2.0, many websites have appeared that allow people to share sound clips online. Some examples are Freesound (http: //freesound.org) or Looperman (http://looperman.com). Soundsnap (http: //soundsnap.com) has followed a similar trend looking for user contributions, but trying a revenue sharing business model. Other sites, such as Indaba(http: //indabamusic.com), Soundcloud (http://soundcloud.com), or ccmixter (http: //ccmixter.org) have incorporated similar functionality to support their musicrelated functionality. One typically essential feature in these systems is the forum. In the forum it is common to see requests for specific recordings, as well as discussions about different techniques, licensing issues and so on.

Audio clip sharing websites offer an interesting example of a collective process that produces a value that goes beyond individuals. The motivations that lead people to record and upload sounds for free are clearly related to social factors. In this paper, we propose a formal analysis of the elements that compose audio clip sharing systems that enables us to better understand this process. The notion of creativity, as a concept that involves both "innovation" and "relevance", can serve as a measure of the success of this process. We hypothesize that the analysis of the different user networks extracted from the information systems can give an insight on how this collective creative process unfolds, and test these ideas analyzing data from Freesound.org, one of the most popular audio clip sharing sites. The rest of this paper is organized as follows. In section 2 we introduce the notion of collective creativity and describe the general hypothesis that it is influenced by the interconnections between users of the system. In section 3 we characterize audio clip sharing systems and the networks of users that can be extracted from them. In section 4 we present an analysis of Freesound.org. We discuss our findings in section 5 .

\section{Collective creativity and small world networks}

\subsection{Creativity and audio clip sharing}

Creativity is usually defined as human ability to "create" things that are new and have some sort of relevance. Beyond this definition there is generally little agreement. A general trend is to consider at least two levels (personal and historical creativity) at which novelty and relevance are evaluated. Personal creativity refers to ideas that are new and interesting to their author, while historical creativity refers to ideas or products that are considered innovative by a larger audience (Boden, 2003). While the traditional discourse focuses on individuals and their mental processes, a certain trend has stressed the social dimension of creativity (Csikszentmihalyi and Wolfe, 2000, Montuori and Purser, 1995).

The notion of creativity can help understanding the build-up of a collective audio clip database. This is quite clear in the case of a very focused and at the same time open community like Freesound.org. Most users are interested in downloading sound files. Only a few consider uploading their own. For these users, 
the game consists in uploading something interesting and new. If the database contains already lots of bass drum sounds, uploading a new one may not seem necessary or especially rewarding. Thus, the process seems to become an exploration of the specific affordance and limits of sharing sound samples, and the site ends up containing a wide diversity of sounds. In this context, creativity can be thought as an attribute of an artifact, in this case an audio recording. Since the creative community of people who record and upload sounds is surrounded by a much larger number of people who only downloads (a typical situation for internet content) the creativity of the sounds, as judged by this larger audience, can be used as a measure of success of the community.

\subsection{Small world networks}

Graph theory in general, and complex networks theory in particular, has received a growing attention from the scientific community in the last decade. Graphs are simple mathematical objects, composed by a collection of vertices (also called nodes), pairwise connected by edges (links). These simple models can be used to represent many problems of practical interest, and help unveiling non-trivial patterns of connections between the elements of any kind of real or virtual systems. Some examples span from biological (Sporns et al., 2004, Tong et al., 2004), social (Barabási et al., 2002), up to technological networks (Guimerà et al., 2005).

Small world networks were formally defined by Watts and Strogatz (Watts and Strogatz, 1998) who characterized them as a crossover between a regular lattice and a random graph. Their model captured a property of many real world networks, where the average distance between any two nodes tends to be as low as in a random graph, while the clustering coefficient (defined as the average of fraction of nodes that are connected among all neighbors of two given nodes) is much higher.

\subsection{The hypothesis of creativity in small worlds}

A common hypothesis is that small world network structures favor creativity and innovation (Cowan and Jonard, 2003). This idea has been tested empirically in different domains: (Verspagen and Duysters, 2004, Uzzi and Spiro, 2005; Fleming, King and Juda, 2007). The general assumption when judging the small world structure is that short path lengths benefit innovation by allowing the flow of information. The effects of high clustering are less obvious. Clustering is generally associated with the flow of redundant information, but also to grouping of similar agents which may improve collaboration (Cowan and Jonard, 2003). Uzzi and Spiro (Uzzi and Spiro, 2005) consider both short path lengths and clustering to impact creativity following an $U$ shape, in the sense that both properties are beneficial up to a certain point, from which they become detrimental due to uniformization. Here, clustering is associated to the formation of conventions, and hence more related to the relevance than to the innovation component of creativity. On the other hand, Fleming, King and Juda (Fleming, King and Juda, 2007) found no significant effect of clustering, and discussed the potential detrimental effects of clustering on innovation. A similar trend is observed for the interaction between high clustering and short path length, which is only shown to have an impact when clustering is relevant. 
These ideas are not necessarily applicable to information networks. Internet applications such as our object of study, allow much faster flows of information, but at the same time, relationships established online are typically considered weaker than real world social ties (Cummings, Butler and Kraut, 2002). In the specific case of audio clip sharing sites, there are no explicit social networks. Still there are many ties that can be defined among users that reflect the relationships and information flow between them. Our main research question is which of these networks, if any, are useful to predict creativity, and how the measures that define small networks exert an impact upon it. This information should help understanding the dynamics of audio clip sharing systems and improve their design.

\section{Audio Clip sharing systems}

\subsection{General structure}

The three main entities that are stored in repositories of audio clips are audio files, users, and textual descriptions of audio files. Most sites use free tags as textual descriptions of the audio content. This structure is very similar, although not equal, to general tagging systems, which are traditionally studied as tripartite hypergraphs (Mika, 2005). In tagging systems, an annotation is seen as a link between a user, a resource and a label. From this representation, several networks can be extracted, for example between users on the basis of their shared resources or on the basis of shared tags. Audio clip sharing sites are different in that users normally describe the files they create and not those of other users. For example in Freesound, tags are mainly generated as part of the file uploading process and, while it is possible for any user to tag sounds, $99 \%$ of the tags are assigned by the author of a sound. A possible explanation may be that, while in tagging sites such as delicio.us or music radios like last.fm users act as active receivers of information and their main activity is consuming and describing that information, in audio clip sharing there is a more practical motivation in using these files (i.e, the creation of music or audiovisual content) and less in directly enjoying them online. A number of implicit networks can be extracted from this activity by considering users as nodes. Following the idea that the community motivates and influences users in their contributions, we hypothesize that these networks contain valuable information in order to understand the dynamics of audio clip file sharing.

\subsection{Implicit networks}

\subsubsection{Downloads network}

We define the download network of an audio clip sharing site as a graph where each node is a user and each edge represents a file that has been downloaded between two users. A user may download many files from the same other user, and thus the network is a multigraph. This network can be seen as an analogy to directed citation networks (White, Wellman and Nazer, 2004). However, for consistency with the rest of networks and with most of the small world literature, we convert this graph to an undirected one. In this case, downloads between two users in both directions 
become a number of undirected edges that represent the strength of the similarity between both users.

\subsubsection{Semantic network}

The semantic network of users of an audio clip sharing site can be derived from the concepts they employ to describe their sounds. In this case, nodes are also users, but edges represent the similarity between two users based on their tags. Thus, if we represent a user with the vector $v_{i}=v_{i 0} \ldots v_{i n}$ where $v_{i j}$ is the number of files that the user $i$ has annotated with tag $j$, the adjacency matrix can be defined using cosine similarity:

$$
a_{i, j}=\frac{v_{i} \cdot v_{j}}{\left\|v_{i}\right\|\left\|v_{j}\right\|},
$$

This network can also be seen as a multigraph by considering a minimum weight unit (Newman, 2004) so that similarity is expressed in integer values.

\subsubsection{Shared interest network}

Another network can be extracted by counting the number of sounds that two users have downloaded in common. This type of co-occurrence tie can be thought of as an analogy to tagging systems (Mika, 2005). A user is represented by a sparse vector that represents the sounds she or he has downloaded. We define this as the shared interest network by computing cosine distances between these vectors.

\subsubsection{Forum network}

Yet another way to represent the community can be made using the activity in the Forum. Forums are a common addition to online community sites for sharing content. We follow standard practice by linking users that have participated in the same threads. Hence again cosine similarity can be used using the vector of threads.

\subsection{Network properties}

Several properties can be used to characterize the described networks, which are commonly used for small world networks. We now describe some of the properties that can be related to creativity measures.

\subsubsection{Density}

The density of a network measures the number of links with respect to the amount of possible links given by the number of nodes. Thus it is simply defined as

$$
D=\frac{2 m_{d}}{n(n-1)}
$$

where $m_{d}$ is the mean degree and $n$ the number of nodes. Thus, density reflects the level of activity in the network, regardless of the number of nodes. 


\subsubsection{Assortativity}

Assortative mixing refers to the tendency of nodes to connect to similar nodes, which can be measured by different criteria (Newman, 2003). One common measure is degree correlation, this is, the Pearson correlation of node degrees. In weighted networks, another possible measure is strength correlation, where strength is the sum of the weights of the edges connected to a node.

\subsubsection{Efficiency}

The traditional small world network model defines that the average shortest path length is similar to the random graph. The average shortest path length measures the minimum number of edges that join any two nodes in the network,

$$
S P L=\frac{1}{n(n-1)} \sum_{i, j} d\left(v_{i}, v_{j}\right)
$$

where $d\left(v_{i}, v_{j}\right)$ is the length of the shortest path between nodes $v_{i}$ and $v_{j}$. The problem with this property is that when the network includes disconnected components, the distance between disconnected nodes is theoretically infinite. A solution is to compute Efficiency, defined as the average of the inverse of the shortest path lengths:

$$
E f f=\frac{1}{n(n-1)} \sum_{i, j} \frac{1}{d\left(v_{i}, v_{j}\right)} .
$$

When no path exists between two nodes, the efficiency is simply zero. Efficiency obviously grows with the number of edges of a network. In order to determine the significance of a certain efficiency value, it must be compared to an equivalent random graph. This graph is usually built according to the Erdős-Rnyi (ER) model, which randomly wires a specified number of nodes given a probability $p$. Following the standard practice (Humphries and Gurney, 2008) with the average shortest path length, we compute the ratio of efficiency with that of the random graph:

$$
E f f_{r}=\frac{E f f}{E f f_{\text {rand }}} .
$$

\subsubsection{Clustering coefficient}

In the original model by Watts and Strogatz, the clustering coefficient was computed as the average fraction of neighbors of a vertex that are connected between them. This is now usually known as the local clustering coefficient. For undirected graphs this is defined as

$$
C_{i}=\frac{2 E_{i}}{k_{i}\left(k_{i}-1\right)}
$$

where $k_{i}$ is the degree of node $i$, and $E_{i}$ counts the number of edges between the nodes connected to node $i$. The clustering of the network is then computed as the average of the local clustering for all nodes. Like in the case of efficiency, we compute the ratio $C C_{r}$ with the equivalent $E R$ graph. 


\subsubsection{Small world coefficient}

Clustering and average path length are usually combined to give a measure of "small-worldness", defined as the quotient of $\frac{C C}{S P L}$. Here we follow the convention but using efficiency, so we define the small world coefficient as

$S W C=C C_{r} E f f_{r}$

\subsubsection{Modularity}

Modularity evaluates a given partition of the network by counting the number of links between nodes in the same partition compared to the number of links that would naturally exist due to their degree. Here we consider modularity of an undirected multigraph, which can be used for the two described networks. Given

the adjacency matrix $A$ of a graph where $A_{i j}$ is the number of links between nodes $i$ and $j$, modularity is defined as

$$
Q=\frac{1}{2 m} \sum_{i j}\left[A_{i j}-\frac{k_{i} k_{j}}{2 m}\right] \delta\left(v_{i} v_{j}\right)
$$

where $m$ is the total number of edges in the network, and $\delta\left(v_{i} v_{j}\right)$ is a function that returns 1 if the group of node $i$ and node $j$ are the same and 0 otherwise.

Given this definition, many algorithms have been described for partitioning a network by directly optimizing modularity. One established method was proposed in (Blondel et al. 2008) that works for undirected weighted networks (multigraphs) and is currently used in a number of network analysis packages. The algorithm works as follows: each node in the network is initially assigned its own community. Then each iteration is divided in two phases. In the first one, each node is moved to its neighboring community that produces the highest modularity. In the second phase, communities are aggregated into nodes. The iterations continue until no further gain in modularity is obtained. This process typically produces a dendrogram that allows the network to be analyzed as a hierarchy of communities.

\subsection{Network dynamics}

One problem with traditional social network analysis is that a single network only captures a given model in a world of changing social ties. In the case of audio clip sharing sites, the network can change significantly over time as new users register and upload more files, while other users stop visiting the site. In order to capture the interactions of active users, we consider a dynamic approach where we extract the networks using only information about events happening in a given time period. In the case of the downloads network, this sample will include users that have uploaded sounds but not logged in that time frame. For the rest of networks, only active users appear as nodes in the network. This contrasts with previous analysis of these networks where we have statically considered the networks accumulated up to a certain point in time(Roma and Herrera, 2010). For the purposes of this paper, our experiments with "accumulated" networks (where all past events are considered at each sample) yielded similar results at much higher computational 
cost, but the extracted measures suffered from high multicollinearity, which made them unfeasible for regression analysis.

\subsection{Creativity measures}

As described, the concept of creativity can be helpful in understanding online behavior in online audio clip sharing. This notion is commonly considered to be composed of a novelty aspect and a relevance aspect. These properties can be attributed to creative artifacts in the context of a given community. One sound can be more novel and/or relevant for the community regardless of who has uploaded it, and the same individuals can be more or less creative depending on the moment and the community who judges their creations. For accounting the novelty component, in the next section we consider an automatic approach that objectively describes the originality of a given sound. Since no user is assumed to have listened to all of the database, it would be difficult for anyone to judge the originality of a sound for the whole community. With respect to the relevance of a sound, we use feedback measures provided by the community.

\subsubsection{Novelty measure}

The issue of determining the novelty of a document has been considered in text retrieval (Tsai, 2010). A simple approach to document level assessment of novelty is to simply consider the distance to the closest neighbor of a document. We adapt this measure to the audio domain by using standard Mel Frequency Cepstrum Coefficients (MFCC). MFCCs are widely used in audio retrieval as generic descriptors of the sound spectrum shape, being a compact way to formally describe different timbre sensations corresponding to different sounds (Rabiner and Juang, 1993). They are extracted by mapping the audio spectrum to the perceptually-inspired Mel scale. The cepstral coefficients are obtained by computing the Discrete Cosine Transform over the log of the mel-filtered spectrum. This gives 13 coefficients that are extracted over a moving window of $20 \mathrm{~ms}$ with an overlap of $11 \mathrm{~ms}$ and averaged over time. A sound is then represented by a vector of 26 features that represent the mean and variance of the MFCCs. This allows us to compute the originality of a sound in a dataset as the distance to its closest neighbor. In order to obtain a novelty value for a given time frame, we use a KD-tree data structure (Bentley, 1975), where we add all sounds of the first time frame. For each subsequent time frame, we average the distance of each sound introduced in that time frame with the closest one in the tree. This average gives us an estimate of the amount of novel information introduced in the database for that period. After all sounds in a given time frame have been processed, they are added to the tree ad the next time frame is processed.

\subsubsection{Relevance measures}

A common feature in web applications is to allow users to participate at least in the form of comments and ratings. Depending on the sites, some of these functionalities may be available. On the other hand, an objective measure of the success of a sound is the number of times it has been downloaded. In the case of comments and ratings, the situation may vary. In our test case, comments and ratings are 
sparse and always very positive, so their mere presence is always an indicator of value of a sound. Thus, we use three measures: number of downloads, number of comments and number of ratings as indicators of the relevance of a clip to the community. Since the number of people who download, comment or rate sounds is much larger (by a factor of 500) than that of the users who upload sounds we can consider these measures as measures of the success of the community of uploaders among their audience. The value for a given time frame is obtained in the following way: for the sounds that are added in that time frame, we consider the number of downloads, comments and ratings that the sound gets during its life since then until the last date of the analysis. Since the probability of getting feedback and downloads increases with the age of a sound, we normalize these values by the number of days that the sound has been in the database. We then average for all sounds in the time frame to obtain an indicator of the creative performance of the community for that month.

\section{Analysis of Freesound.org}

In order to analyze the properties of the different networks and their effect on the creativity measures, we computed all the described properties for the data in Freesound.org, one of the most popular audio clip sharing communities, over a period of 66 months, from April 2005 to October 2010. Table 1 shows the mean and standard deviations of the different properties of the networks. All of the described networks exhibit similar characteristics. With a few exceptions, they have negative assortativity in both degree and strength, which is a common characteristic of information networks. Also with one exception (the shared interest network) they have high modularity, above the empirical threshold of 0.3 commonly considered to indicate the presence of modular structure (Newman and Girvan, 2004). The clustering coefficient ratios show that the networks tend to be much more clustered than the equivalent random graph, while the efficiencies are similar to random graphs, and so the efficiency ratios revolve around 1. Hence, the small world coefficients (the product of efficiency and clustering) generally follow the clustering coefficient. This reflects that the measured networks follow the small-world network model. We now analyze how these variables are related to the creativity measures described above through regression analysis. Since the evolution of small world coefficient is strongly tied to the clustering coefficient, and it does not make sense to included it in the same models as its factors, we omit this property from further analysis.

\subsection{Regression analysis}

In order to test the influence of the small world properties of all four networks on the creativity measures we performed ordinary least squares regression analysis with the described variables for each of the networks. Our aim is to understand to which extent the properties of each network can model the creative outcome of the community. In order to account for potential causal relationships, we introduce a lag of one time period between the predictors and the dependent variables. For each model, we checked the variance inflation factor (VIF) to ensure that there are 
Table 1 Mean (standard deviation) of the described properties for the different networks: degree correlation (dc), strength correlation (sc), modularity (m), clustering coefficient ratio(cc), efficiency ratio(ef), small world coefficient (swc) and density (den)

\begin{tabular}{|r|r|r|r|r|}
\hline \hline Variable & Downloads & Semantic & Shared & Forum \\
\hline \hline dc & $-0.13(0.05)$ & $-0.17(0.06)$ & $0.33(0.31)$ & $-0.22(0.15)$ \\
sc & $-0.01(0.00)$ & $-0.01(0.01)$ & $-0.01(0.02)$ & $0.04(0.10)$ \\
m & $0.45(0.06)$ & $0.40(0.05)$ & $0.17(0.16)$ & $0.55(0.16)$ \\
cc & $5.17(1.52)$ & $3.33(0.73)$ & $7.31(2.55)$ & $4.87(2.07)$ \\
ef & $1.14(0.05)$ & $0.97(0.02)$ & $0.85(0.08)$ & $0.71(0.16)$ \\
swc & $5.91(1.86)$ & $3.22(0.68)$ & $6.19(2.16)$ & $3.66(2.19)$ \\
den & $0.01(0.02)$ & $0.21(0.05)$ & $0.09(0.05)$ & $0.10(0.14)$ \\
\hline
\end{tabular}

Table 2 Regression analysis coefficients for the downloads network. Significance codes: $*(p<0.5),{ }^{* *}(p<0.1),{ }^{* * *}(p<0.01)$

\begin{tabular}{|c|c|c|c|c|}
\hline \hline Variable & Novelty & Downloads & Ratings & Comments \\
\hline \hline $\mathrm{dc}$ & $-26.36^{*}$ & -0.08 & -0.01 & -0.00 \\
$\mathrm{sc}$ & -62.72 & $8.09^{* *}$ & $0.29^{* *}$ & $0.05^{* *}$ \\
$\mathrm{~m}$ & -20.01 & $0.53^{* * *}$ & $0.02^{* * *}$ & $0.00^{* *}$ \\
$\mathrm{cc}$ & 0.41 & $0.002^{* * *}$ & 0.00 & $0.00^{* *}$ \\
$\mathrm{ef}$ & 7.44 & 0.008 & 0.00 & 0.00 \\
$\mathrm{den}$ & $450.05^{* * *}$ & $5.86^{* * *}$ & $0.15^{* * *}$ & $0.03^{* * *}$ \\
\hline \hline$R^{2}$ & 0.85 & 0.41 & 0.25 & 0.19 \\
\hline
\end{tabular}

Table 3 Regression analysis coefficients for the semantic network.

\begin{tabular}{|c|c|c|c|c|}
\hline \hline Variable & Novelty & Downloads & Ratings & Comments \\
\hline \hline $\mathrm{dc}$ & $-38.18^{* * *}$ & $-0.24^{*}$ & -0.00 & -0.00 \\
$\mathrm{sc}$ & $-731.71^{* * *}$ & $2.92^{* *}$ & 0.15 & 0.02 \\
$\mathrm{~m}$ & $20.82^{*}$ & 0.20 & 0.01 & -0.00 \\
$\mathrm{cc}$ & $3.78^{*}$ & $-0.00^{* *}$ & -0.00 & -0.00 \\
$\mathrm{ef}$ & $-15.07^{* * *}$ & $-1.48^{* *}$ & -0.05 & -0.00 \\
$\mathrm{den}$ & $111.23^{* * *}$ & $1.51^{* *}$ & 0.04 & 0.00 \\
\hline \hline$R^{2}$ & $0.76^{2}$ & $0.49^{*}$ & 0.36 & 0.09 \\
\hline
\end{tabular}

no collinearity problems. All of the VIF values were below 10, which is the usually recommended threshold (Hair et al. 2010) with the single exception of modularity in the shared interest network which went up to 10.8 .

Tables 2-5 show the main regression data for predicting either novelty, downloads, ratings or comments, using the 4 different networks we have studied (downloads, semantic, shared interest and forum). For each dependent variable, the coefficients of the predictors are listed. Sign indicates the direction of the relationship (direct or inverse) and high absolute values indicate that the factor is relevant to predict that target variable. Statistical significance is indicated with the usual asterisk coding (see caption for details). The bottom cell presents the determination coefficient $\left(R^{2}\right)$ which indicates the predictive accuracy of each regression function. 
Table 4 Regression analysis coefficients for the shared interest network.

\begin{tabular}{|c|c|c|c|c|}
\hline \hline Variable & Novelty & Downloads & Ratings & Comments \\
\hline \hline $\mathrm{dc}$ & 1.50 & -0.11 & -0.00 & -0.00 \\
$\mathrm{sc}$ & -87.23 & $-1.91^{*}$ & -0.02 & 0.00 \\
$\mathrm{~m}$ & $20.08^{*}$ & -0.24 & -0.01 & -0.00 \\
$\mathrm{cc}$ & -0.03 & -0.01 & -0.00 & 0.00 \\
$\mathrm{ef}$ & -5.11 & 0.18 & 0.01 & -0.00 \\
$\mathrm{den}$ & $114.30^{* *}$ & -0.28 & -0.01 & 0.00 \\
\hline \hline$R^{2}$ & 0.76 & 0.40 & 0.24 & 0.05 \\
\hline
\end{tabular}

Table 5 Regression analysis coefficients for the forum network.

\begin{tabular}{|c|c|c|c|c|}
\hline Variable & Novelty & Downloads & Ratings & Comments \\
\hline \hline $\mathrm{dc}$ & $-21.45^{* * *}$ & $-0.17^{* *}$ & -0.00 & -0.00 \\
$\mathrm{sc}$ & $32.11^{* *}$ & $0.04^{* *}$ & 0.00 & -0.00 \\
$\mathrm{~m}$ & $-6.97^{*}$ & $-0.20^{* *}$ & -0.01 & -0.00 \\
$\mathrm{cc}$ & $-0.92^{*}$ & $-0.01^{*}$ & -0.00 & -0.00 \\
$\mathrm{ef}$ & $4.06^{* * *}$ & -0.00 & -0.00 & -0.00 \\
$\mathrm{den}$ & $52.69^{* * *}$ & -0.19 & -0.01 & -0.00 \\
\hline \hline$R^{2}$ & 0.60 & 0.36 & 0.27 & 0.05 \\
\hline
\end{tabular}

\section{Discussion}

The results show similar patterns with respect to the accuracy of the models, as measured by $R^{2}$. Novelty can be predicted with better accuracy in most of the networks. This seems more understandable if one takes into account that novelty is calculated using information from the same time period (except for the introduced lag) than the predictors. The rest of variables count the numbers of downloads, ratings and comments that sounds have received over time, which is a rough indicator of their quality but can obviously be influenced by a number of external factors. On the other hand, network density appears to be a generally dominant factor.

\subsection{Novelty}

The downloads network shows the highest accuracy with respect to the novelty variable, explaining almost $85 \%$ of its variance. The most important factor in this model is network density. This indicates that a higher number of downloading interactions between users may have a positive effect on innovation. However, in the case of novelty, there is no reason to think that such potential causality would not go in the opposite direction. On the other hand, degree correlation has a negative impact, which could indicate that interactions among different users (in terms of degree) are positively related to novelty. This tendency is reinforced in those of the other networks that best predict novelty. In the semantic network model, the strength correlation appears also as a negative significant factor. Modularity and clustering coefficient appear as small positive contributions in the semantic and shared networks. On the contrary, the effect of clustering of forum posts seems to be 
negative. In summary, novelty of uploaded sounds seems to be positively correlated with high connectivity, in some cases with a small bias towards clustering, and disassortative mixing.

\subsection{Relevance}

With respect to the relevance measures, the semantic network seems to have the greatest predictive power, followed by networks based on downloads (downloads and shared interest). For example almost $50 \%$ of the variance in the average number of downloads of a sound can be explained by the semantic network properties. In all of the networks, strength correlation appears along with density as an important positive factor. Also noticeable are the significant correlations of modularity and clustering in the downloads network. In contrast, degree correlation has a significant negative contribution in the semantic and also in the forum networks. This seems to point towards connectivity among active users (who should have higher strength regardless of the degree) as a factor for the relevance measures. Particularly in the semantic network, strength reflects the level of agreement of a user with other users in the tags that they use for the sounds. This describes an important connection between the people who upload sounds, which will affect the findability of sounds in the database. In contrast, efficiency (i.e. the harmonic mean of the average shortest path length) in the semantic network has a negative effect, which could mean that connecting remote users, who could be interested in different topics, with respect to text descriptions, is not beneficial. In conclusion, relevance seems to be also very related to network density in general and slightly with local density. Moreover, the agreement between users when describing sounds, reflected by strength correlation and density of the semantic network, seems to be among the most important factors for predicting relevance.

\subsection{Relation to the small world hypotheses}

While the studied networks have shown to follow the small-world network model, our results show some differences with respect to the hypotheses reviewed in section 2.3. The referenced works all analyze physical networks, which may follow different rules than networks mediated by information technology. In real world social networks, connections between distant clusters seem more difficult to achieve and therefore more valuable for diffusing information. In contrast, in internetbased communities, finding different people is very usual. In these communities, the difficulty lies in finding people with similar interests among the variety. In the context of the Freesound community this seems a reasonable explanation, since the site poses no restrictions or biases with respect to the kinds of sounds that can be uploaded, which results in a melting pot of many different cultures. Given network density as a general factor for the creative outcome, it would seem that local density and clustering, as reflected by the clustering coefficient ratio and modularity, are to be preferred to short path lengths. Our study also points towards the importance of assortative mixing, which is not usually covered in the small world creativity literature. An interesting pattern in the analyzed data is that, while degree correlation (i.e. assortative mixing among nodes of similar number of connections to different users) is negatively correlated to creativity measures, 
strength correlation (which better reflects the level of activity of users in valued networks)

\section{Conclusions}

It is often difficult to define the goals of a community based system, where each user may have different motivations. This makes it difficult to design and develop software for community usage. For the case of audio clip sharing, we have proposed a set of indicators of the creative outcome of the active community (users who create, or record, and upload sounds) using data from the larger community of information consumers. We have hypothesized that these measures partly depend on the dynamics of the community, which can be measured using the different networks of interactions in a given time frame. Our results show that part of the variance of these measures can in fact be explained by network measures. The proposed method, as well as the results of our empirical study, can be used to improve the design and functionalities of audio clip sharing sites. In this sense, it seems that features that try to maximize the density of the different networks, i.e. by facilitating actions that connect users, would impact on the quality and novelty of sounds that are uploaded. Similarly features that promoting connectivity among most active users, which would reflect on strength correlation, and features that promote clustering of users with similar interests are to be preferred.

As future work, the relationship between assortative mixing and creativity could be further explored using other measures of similarity between users (beyond degree and strength), such as similarities between the sounds they download. Another possibility is analyzing the outcomes of individuals or smaller communities and their relationship to their network positions and centralities.

\section{References}

Barabási, A.L., H. Jeong, Z. Néda, E. Ravasz, A. Schubert and T. Vicsek. 2002. "Evolution of the social network of scientific collaborations." Physica A: Statistical Mechanics and its Applications 311(3-4):590-614.

Bentley, J.L. 1975. "Multidimensional binary search trees used for associative searching." Communications of the ACM 18:509-517.

Blondel, V.D., J.-L. Guillaume, R. Lambiotte and E. Lefebvre. 2008. "Fast unfolding of community hierarchies in large networks." Journal of Statistical Mechanics 10:17425468 .

Boden, M. 2003. The Creative Mind: Myths and Mechanisms. 2nd ed. London: Routledge.

Cowan, R. and N. Jonard. 2003. "The dynamics of collective invention." Journal of Economic Behavior \& Organization 52(4):513-532.

Csikszentmihalyi, M. and R. N. Wolfe. 2000. New conceptions and research approaches to creativity: Implications of a systems perspective for creativity in education. In International handbook of giftedness and talent, ed. K. Heller, F. Monks, R. Subotnik and R. Sternberg. 2nd ed. Oxford: Pergamon Press pp. 81-92.

Cummings, J.N., B. Butler and R. Kraut. 2002. "The quality of online social relationships." Communications of the ACM 45:103-108. 
Fleming, L., C. King and A.I. Juda. 2007. "Small Worlds and Regional Innovation." Organization Science 18(6):938-954.

Guimerà, R., S. Mossa, A. Turtschi and L.A.N. Amaral. 2005. "The worldwide air transportation network: Anomalous centrality, community structure, and cities' global roles." Proceedings of the National Academy of Sciences of the United States of America 102(22):7794-7799.

Hair, J.F., W.C. Black, B.J. Babin and R.E. Anderson. 2010. Multivariate data analysis: a global perspective. 7th ed. London: Pearson Education.

Humphries, M.D. and K. Gurney. 2008. "Network 'Small-World-Ness': A Quantitative Method for Determining Canonical Network Equivalence." PLoS ONE 3(4):e0002051.

Lessig, L. 2008. Remix: making art and commerce thrive in the hybrid economy. New York: Penguin Press.

Mika, P. 2005. Ontologies Are Us: A unified model of social networks and semantics. In Proceedings of the 4 th International Semantic Web Conference. Galway, Ireland: Springer pp. 522-536.

Montuori, A. and R.E. Purser. 1995. "Deconstructing the Lone Genius Myth: Toward a Contextual View of Creativity." Journal of Humanistic Psychology 35(3):69-112.

Newman, M. E. J. and M. Girvan. 2004. "Finding and evaluating community structure in networks." Physical Review E 69(2):026113.

Newman, M.E.J. 2003. "Mixing patterns in networks." Physical Review E 67:026126.

Newman, M.E.J. 2004. "Analysis of weighted networks." Physical Review E 70(5):056131.

Rabiner, L. and B.H. Juang. 1993. Fundamentals of Speech Recognition. 1st ed. Englewood Cliffs: Prentice Hall.

Roma, G. and P. Herrera. 2010. Community Structure in Audio Clip Sharing. In Proceedings of the 2010 International Conference on Intelligent Networking and Collaborative Systems. Thessaloniki, Greece: IEEE Computer Society pp. 200-205.

Sporns, O., D.R. Chialvo, M. Kaiser and C.C. Hilgetag. 2004. "Organization, development and function of complex brain networks." Trends in Cognitive Sciences 8(9):418-425.

Tong, A.H.Y., G. Lesage, G.D. Bader, H. Ding, H. Xu, X. Xin, J. Young, G.F. Berriz, R.L. Brost, M. Chang, Y. Chen, X. Cheng, G. Chua, H. Friesen, D.S. Goldberg, J. Haynes, C. Humphries, G. He, S. Hussein, L. Ke, N. Krogan, Z. Li, J.N. Levinson, H. Lu, P. Menard, C. Munyana, A.B. Parsons, O. Ryan, R. Tonikian, T. Roberts, A.-M. Sdicu, J. Shapiro, B. Sheikh, B. Suter, S.L. Wong, L.V. Zhang, H. Zhu, C.G. Burd, S. Munro, C. Sander, J. Rine, J. Greenblatt, M. Peter, A. Bretscher, G. Bell, F.P. Roth, G.W. Brown, B. Andrews, H. Bussey and C. Boone. 2004. "Global Mapping of the Yeast Genetic Interaction Network." Science 303:808-813.

Tsai, F.S. 2010. "Review of Techniques for Intelligent Novelty Mining." Information Technology Journal 9(6):1255-1261.

Uzzi, B. and J. Spiro. 2005. "Collaboration and Creativity: The Small World Problem." American Journal of Sociology 111(2):447-504.

Verspagen, B. and G. Duysters. 2004. "The small worlds of strategic technology alliances." Technovation 24(7):563-571.

Watts, D.J. and S.H. Strogatz. 1998. "Collective dynamics of 'small-world' networks." Nature 393(6684):440-442.

White, H.D., B. Wellman and N. Nazer. 2004. "Does citation reflect social structure?: longitudinal evidence from the "Globenet" interdisciplinary research group." Journal of the American Society for Information Science and Technology 55(2):111-126. 